\title{
BMJ Open Long-term effects of adenotonsillectomy in children with obstructive sleep apnoea: protocol for a systematic review
}

\author{
Felipe Damiani, ${ }^{1}$ Gabriel Rada, ${ }^{2,3,4}$ Juan Cristóbal Gana, ${ }^{5}$ Pablo E Brockmann, ${ }^{6,7}$ \\ Gigliola Alberti ${ }^{5}$
}

To cite: Damiani F, Rada G, Gana JC, et al. Long-term effects of adenotonsillectomy in children with obstructive sleep apnoea: protocol for a systematic review. BMJ Open 2016;6: 010030 .

doi:10.1136/bmjopen-2015010030

- Prepublication history for this paper is available online. To view these files please visit the journal online (http://dx.doi.org/10.1136/ bmjopen-2015-010030)

Received 17 September 2015 Revised 12 February 2016 Accepted 23 February 2016

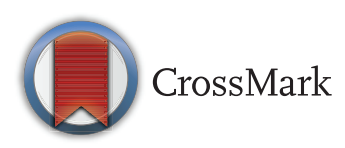

For numbered affiliations see end of article.

\section{Correspondence to} Dr Gigliola Alberti; galberti@med.puc.cl

\section{ABSTRACT}

Introduction: Adenotonsillar hypertrophy is the most important anatomical factor associated with obstructive sleep apnoea syndrome in children. The American Academy of Pediatrics recommends adenotonsillectomy as the first line of treatment. AT can reduce the apnoea hypopnoea index; however, its effect on long-term outcomes remains unclear.

Methods and analysis: We will conduct an electronic search for randomised controlled trials in MEDLINE, Cochrane Central Register of Controlled Trials (CENTRAL) and EMBASE. We will also identify literature by reviewing the references included in the selected studies and relevant reviews, screening through important scientific conferences, and searching for ongoing trials in the WHO International Clinical Trials Registry Platform. Two researchers will independently undertake selection of studies, data extraction and assessment of the risk of bias of included studies. We will estimate pooled risk ratios for dichotomous data, and mean difference or standardised mean difference for continuous outcomes. A random-effects model will be used for meta-analyses. Data synthesis and other analyses will be conducted using RevMan V.5.3 software.

Ethics and dissemination: No ethics approval is considered necessary. The results of this study will be disseminated via peer-reviewed publications and social networks.

Trial registration number: CRD42015022102.

\section{BACKGROUND}

\section{Description of the condition}

Obstructive sleep apnoea (OSA) is the most severe form of sleep-disordered breathing. It includes episodes of increased upper airway resistance and respiratory effort with partial or complete upper airway obstruction and combinations of snoring, intermittent hypoxaemia, hypercarbia and awakenings. ${ }^{1}{ }^{2}$ In children, OSA occurs at different ages and its prevalence ranges from $0.7 \%$ to $10 \%$ with a peak between 2 and 8 years. ${ }^{3}$ This syndrome is associated with several adverse

\section{Strengths and limitations of this study}

- The main goals of this review are to contribute with more robust evidence regarding the effects of adenotonsillectomy on quality of life and longterm outcomes in children with obstructive sleep apnoea, and to provide high-quality evidence that may be useful for patients and clinicians.

- The main limitation might result from the reduced number of primary studies.

outcomes, including cognitive impairment, metabolic and cardiovascular consequences, behavioural deficits ${ }^{4-6}$ and lower quality of life (QoL). ${ }^{7}$ The pathophysiological factors implicated in childhood OSA syndrome can be separated in anatomical factors that produce reduction in airway calibre and other factors that promote increased upper airway collapsibility such as obesity and presence of upper airway inflammation. ${ }^{6} 8$ Adenotonsillar hypertrophy is the most important anatomical factor that has been associated with obstructive apnoea. ${ }^{9}$

\section{Description of the intervention}

The American Academy of Pediatrics recommends adenotonsillectomy as the first line of treatment for children with OSA. ${ }^{3} \quad 10 \quad 11$ Adenotonsillectomy is usually a safe and minor procedure but can be associated with significant complications including death. The conventional technique is cold steel and/or electrocautery dissection (CS/EC). Nevertheless, a variety of approaches have been tested over the years. ${ }^{12}$ In general, AT in children has low complication rates, which may include pain, diminished oral intake, bleeding and infection. ${ }^{413}$

How the intervention might work

Removal of the tonsils combined with adenoidectomy eliminates the upper airway obstruction improving the breathing 
pattern. ${ }^{14}$ The effectiveness of AT has been confirmed by several studies that show an improvement in the apnoea hypopnoea index measured by objective parameters. ${ }^{15}{ }^{16}$ Besides, observational studies have suggested that AT improves QoL in paediatric patients with OSA, ${ }^{17} 18$ specifically in areas of general health perception, caregivers' concerns and family life. ${ }^{7}$

\section{Why it is important to do this review}

Until now, there are few reported systematic reviews analysing the long-term effects of adenotonsillectomy in children with sleep-disordered breathing. Previous systematic reviews in this field have reported the impact of AT on sleep apnoea with limited results. ${ }^{19}$ Besides, recent evidence showed controversial results in the QoL comparing only adenotonsillectomy versus adenotonsillotomy and including randomised and non-randomised studies. ${ }^{20}$ Further, the effect of AT in growth, measured through height and weight, has been evaluated by observational studies. ${ }^{21}$ Therefore, it is important to evaluate adenotonsillectomy comprehensively and to provide an up-to-date synthesis of the evidence regarding this question for clinicians and patients, paying especial attention to long-term outcomes.

\section{OBJECTIVES}

To evaluate the effects of adenotonsillectomy in children with OSA.

\section{METHODS}

\section{Criteria for considering studies for this review}

Types of studies

We will include randomised controlled trials (RCTs).

\section{Types of participants}

Participants under 18 years with a diagnosis of OSA based on polysomnographic criteria or subjective measurements will be eligible for inclusion, irrespective of the severity or duration of the disease.

\section{Types of interventions}

Treatment group: tonsillectomy and/or adenoidectomy.

Control group:

- Other medical or mechanical intervention

- Other surgical treatments different to tonsillectomy or adenotonsillectomy

- Watchful or no intervention

Types of outcome measures

Primary outcome:

QoL.

Secondary outcomes:

- All cause mortality

- Change in height and/or weight

- Frequency of apnoea or hypopnoea

- Daytime sleepiness

- Snoring

- Complications of surgery
Searching methods for identification of studies

Electronic searches

We will conduct searches in the Cochrane Central Register of Controlled Trials (CENTRAL), EMBASE and MEDLINE. We will not apply language or publication status restriction.

The search strategy for MEDLINE (PubMed) is the following:

\#1 "Tonsillectomy" [Mesh];

\#2 "Palatine Tonsil/surgery" [Mesh];

\#3 adenotonsillecto* OR adenotonsilectom* OR tonsillecto* OR tonsillotom* OR adenoidectom* OR tonsilotom*;

\#4 (adenotonsil* OR tonsil* OR adenoid* OR "Palatine Tonsil" [Mesh] OR "Adenoids"[Mesh]) AND ("Surgery" [Mesh] OR surger* OR surgic* OR excis* OR extract* OR remov*);

\#5 \#1 OR \#2 OR \#3 OR \#4;

\#6 "Sleep Apnea Syndromes"[Mesh];

\#7 sleep* AND (apnea* OR apnoea*);

\#8 hypopnea* OR hypopnoea*;

\#9 OSA OR OSAHS;

\#10 \#6 OR \#7 OR \#8 OR \#9;

$\# 11$ randomized controlled trial [pt];

\#12 controlled clinical trial [pt];

\#13 randomized [tiab];

\#14 placebo [tiab];

\#15 drug therapy [sh];

$\# 16$ randomly [tiab];

$\# 17$ trial [tiab];

\#18 groups [tiab];

\#19 \#11 OR \#12 OR \#13 OR \#14 OR \#15 OR \#16 OR

\#17 OR \#18;

\#20 animals [mh] NOT humans [mh];

\#21 \#19 NOT \#20;

\#22 \#5 AND \#10 AND \#21.

The MEDLINE strategy will be adapted to the syntax and subject headings of the other databases.

\section{Searching other resources}

Additional studies will be sought by:

- Manually searching the references of included studies and relevant review articles.

- Contacting experts in the field.

- Searching in the WHO International Clinical Trials Registry Platform.

- Screening conference proceedings of the World Sleep Federation, European Respiratory Society and American Thoracic Society during the past 10 years.

\section{Data collection and analysis}

We will follow the guidelines provided in the Cochrane Handbook for Systematic Reviews of Interventions. ${ }^{22}$ Analyses will be performed using Review Manager V.5.3 (Review Manager (RevMan) [Computer program]. Version 5.3. Copenhagen: The Nordic Cochrane Centre, The Cochrane Collaboration, 2014). 


\section{Selection of studies}

All publications will be reviewed independently for eligibility by FD and GA. To be eligible, each publication will be assessed to determine if it meets the inclusion criteria as detailed above. Full-text articles of all potentially eligible abstracts will be evaluated by the same reviewers. Any disagreement will be resolved by consensus, or by a third reviewer if needed (GR).

\section{Data extraction and management}

Two review authors (FD and GA) will independently complete the data extraction form. The following data will be retrieved:

1. General information of the study: title, journal, year, publication status.

2. Study characteristics: sample size, number of arms, methodology of randomisation and allocation concealment, blinding of participants.

3. Participants: age, sex, race, height, weight, body mass index, time from OSA diagnosis, diagnostic criteria and severity of OSA, previous surgeries, comorbid conditions, concomitant medication and others sleep treatments.

4. Interventions: type of surgery, medical treatments, watchful or no intervention.

5. Outcomes: objective or subjective methods, measuring instrument and scale, follow-up time.

6. Results: point estimates and measures of variability, frequency counts for dichotomous variables, number of patients.

\section{Assessment of risk of bias in included studies}

Two review authors (FD and GA) will independently assess the risk of bias of each included trial using the Cochrane Risk of Bias tool according to the recommendations in the Cochrane Handbook for Systematic Reviews of Interventions. ${ }^{22}$ Studies will be judged for having a high, low or unclear risk of bias.

\section{Measures of treatment effect}

For dichotomous variables, we will calculate the risk ratio with $95 \%$ CI. For continuous variables, we will calculate the mean difference (MD) and/or standardised MD with $95 \%$ CI.

\section{Unit of analysis issues}

The unit of analysis will be the individual patient. Since only standard-design RCTs are expected, each patient will have received only one intervention. In studies where more than two interventions were compared, extra care will be taken to avoid including the same patients twice in the meta-analysis.

\section{Dealing with missing data}

If data are missing in a published report, we will contact the original investigators to request missing data.

\section{Assessment of heterogeneity}

To assess heterogeneity between the trials, we will specifically examine the degree of heterogeneity observed in the results with the $\mathrm{I}^{2}$ statistic, where $\mathrm{I}^{2}$ values of $50 \%$ or more indicate a substantial level of heterogeneity. ${ }^{23}$

\section{Assessment of reporting biases}

Reporting biases will be assessed with funnel plots of the relative risk estimates from the individual trials (plotted on a logarithmic scale) against trial size or precision (variance) or the estimators. Funnel plots will be constructed if there are at least 10 included trials.

\section{Data synthesis}

The analyses will be performed in Review Manager V.5.3 (Review Manager (RevMan, 2014)). We will use the random-effects model for all meta-analyses.

\section{Subgroup analysis and investigation of heterogeneity}

We will aim to perform the following subgroup analyses:

- Nutritional status of the patients (obese and not obese);

- Age (infant, preschool and school children);

- OSA diagnostic method (polysomnographically proven OSA vs subjective measurements only).

\section{Sensitivity analysis}

We will undertake sensitivity analyses that may explain our findings as well as any observed heterogeneity. Sensitivity analyses will examine the impact of the following variables on the pooled effect:

- Low risk of bias versus unclear or high risk of bias;

- Relevant loss to follow-up (>10\%): best-case versus worst-case scenario.

\section{Summary of findings tables}

Summary of findings tables will provide information about certainty of the evidence and magnitude of effects. We will create summary of findings tables using GRADEpro (ims.cochrane.org/revman/other-resources/ gradepro).

\section{Author affiliations}

${ }^{1}$ Faculty of Medicine, Pontificia Universidad Católica de Chile

${ }^{2}$ Evidence-Based Healthcare Program, Pontificia Universidad Católica de Chile

${ }^{3}$ Department of Internal Medicine, Pontificia Universidad Católica de Chile

${ }^{4}$ Epistemonikos foundation

${ }^{5}$ Division of Pediatrics, Department of Gastroenterology and Nutrition, School of Medicine, Pontificia Universidad Católica de Chile

${ }^{6}$ Department of Pediatric Cardiology and Pulmonology, School of Medicine, Pontificia Universidad Católica de Chile

${ }^{7}$ Faculty of Medicine, Sleep Medicine Center, Pontificia Universidad Católica de Chile

Twitter Follow Gabriel Rada at @radagabriel

Contributors FD and GA formulated the research question and drafted the protocol. GR provided methodological expert opinion and reviewed the protocol. JCG and PEB provided content expertise.

Funding Article processing charges will be funded by the Department of Public Health, Faculty of Medicine, Pontificia Universidad Católica de Chile. 
Competing interests None declared.

Provenance and peer review Not commissioned; externally peer reviewed.

Data sharing statement The results of this study will be disseminated via peer-reviewed publications and social networks.

Open Access This is an Open Access article distributed in accordance with the Creative Commons Attribution Non Commercial (CC BY-NC 4.0) license, which permits others to distribute, remix, adapt, build upon this work noncommercially, and license their derivative works on different terms, provided the original work is properly cited and the use is non-commercial. See: http:// creativecommons.org/licenses/by-nc/4.0/

\section{REFERENCES}

1. Brockmann PE, Schaefer C, Poets A, et al. Diagnosis of obstructive sleep apnea in children: a systematic review. Sleep Med Rev 2013;17:331-40.

2. Li AM, Zhu Y, Au CT, et al. Natural history of primary snoring in school-aged children: a 4-year follow-up study. Chest 2013;143:729-35.

3. Schechter MS. Technical report: diagnosis and management of childhood obstructive sleep apnea syndrome. Pediatrics 2002;109: e69.

4. Marcus CL, Brooks LJ, Ward SD, et al. Diagnosis and management of childhood obstructive sleep apnea syndrome abstract. Pediatrics 2012;130:e714-55.

5. Verhulst SL, Rooman R, Van Gaal L, et al. Is sleep-disordered breathing an additional risk factor for the metabolic syndrome in obese children and adolescents? Int J Obes (Lond) 2009;33:8-13.

6. Tan HL, Gozal D, Kheirandish-Gozal L. Obstructive sleep apnea in children: a critical update. Nat Sci Sleep 2013;5:109-23.

7. Baldassari CM, Mitchell RB, Schubert C, et al. Pediatric obstructive sleep apnea and quality of life: a meta-analysis. Otolaryngol Head Neck Surg 2008;138:265-73.

8. Dayyat E, Kheirandish-Gozal L, Capdevila O, et al. Obstructive sleep apnea in children: relative contributions of body mass index and adenotonsillar hypertrophy. Chest 2009;136:137-44.

9. Verhulst SL, Schrauwen N, Haentjens D, et al. Sleep-disordered breathing in overweight and obese children and adolescents: prevalence, characteristics and the role of fat distribution. Arch Dis Child 2007;92:205-8.

10. Ingram DG, Friedman NR. Toward adenotonsillectomy in children. JAMA Pediatr 2015;169:1.

11. De Luca Canto G, Pacheco-Pereira C, Aydinoz S, et al. Adenotonsillectomy complications: a meta-analysis. Pediatrics 2015;136:702-18.

12. Alexiou VG, Salazar-Salvia MS, Jervis PN, et al. Modern technology-assisted vs conventional tonsillectomy. Arch Otolaryngol Head Neck Surg 2011;137:558-70.

13. Chaidas KS, Kaditis AG, Papadakis CE, et al. Tonsilloplasty versus tonsillectomy in children with sleep-disordered breathing: short- and long-term outcomes. Laryngoscope 2013;123:1294-9.

14. Koltai PJ, Solares CA, Koempel JA, et al. Intracapsular tonsillar reduction (partial tonsillectomy): reviving a historical procedure for obstructive sleep disordered breathing in children. Otolaryngol Head Neck Surg 2003;129:532-8.

15. Mitchell RB. Adenotonsillectomy for obstructive sleep apnea in children: outcome evaluated by pre- and postoperative polysomnography. Laryngoscope 2007;117:1844-54.

16. Marcus CL, Moore $\mathrm{RH}$, Rosen $\mathrm{CL}$, et al. A randomized trial of adenotonsillectomy for childhood sleep apnea. $N$ Engl J Med 2013;368:2366-76.

17. Mitchell RB, Kelly J, Call E, et al. Quality of life after adenotonsillectomy for obstructive sleep apnea in children. Arch Otolaryngol Head Neck Surg 2004;130:190-4.

18. Flanary VA. Long-term effect of adenotonsillectomy on quality of life in pediatric patients. Laryngoscope 2003;113:1639-44.

19. Lim J, McKean MC. Adenotonsillectomy for obstructive sleep apnoea in children (Review). Cochrane Database Syst Rev 2009;(2): CD003136.

20. Wang $\mathrm{H}, \mathrm{Fu} \mathrm{Y}$, Feng $\mathrm{Y}$, et al. Tonsillectomy versus tonsillotomy for sleep-disordered breathing in children: a meta analysis. PLOS ONE 2015;10:e0121500.

21. Bonuck KA, Freeman K, Henderson J. Growth and growth biomarker changes after adenotonsillectomy: systematic review and meta-analysis. Arch Dis Child 2009;94:83-91.

22. Higgins JPT, Green S, eds. Cochrane handbook for systematic reviews of interventions version 5.1.0 [updated March 2011]. The Cochrane Collaboration, 2011. http://www.cochrane-handbook.org

23. Higgins JP, Thompson SG. Quantifying heterogeneity in a meta-analysis. Stat Med 2002;21:1539-58. 